\title{
A Molecular Tweezer Ameliorates Motor Deficits in Mice Overexpressing $\alpha$-Synuclein
}

\author{
Franziska Richter ${ }^{1} \cdot$ Sudhakar R. Subramaniam ${ }^{1} \cdot$ Iddo Magen $^{1} \cdot$ Patrick Lee $^{1}$ • \\ Jane Hayes $^{1}$ • Aida Attar ${ }^{1,2}$ • Chunni Zhu ${ }^{1}$. Nicholas R. Franich ${ }^{1}$. Nicholas Bove ${ }^{1}$. \\ Krystal De La Rosa ${ }^{1}$ • Jacky Kwong ${ }^{1}$ - Frank-Gerrit Klärner ${ }^{3}$ • Thomas Schrader ${ }^{3}$. \\ Marie-Françoise Chesselet ${ }^{1,2,4,5} \cdot$ Gal Bitan ${ }^{1,2,5}$
}

Published online: 5 June 2017

(C) The American Society for Experimental NeuroTherapeutics, Inc. 2017

\begin{abstract}
Aberrant accumulation and self-assembly of $\alpha$ synuclein are tightly linked to several neurodegenerative diseases called synucleinopathies, including idiopathic Parkinson's disease, dementia with Lewy bodies, and multiple system atrophy. Deposition of fibrillar $\alpha$-synuclein as insoluble inclusions in affected brain cells is a pathological hallmark of synucleinopathies. However, water-soluble $\alpha$-synuclein oligomers may be the actual culprits causing neuronal dysfunction and degeneration in synucleinopathies. Accordingly, therapeutic approaches targeting the toxic $\alpha$ synuclein assemblies are attractive for these incurable disorders. The "molecular tweezer" CLR01 selectively remodels abnormal protein self-assembly through reversible binding to Lys residues. Here, we treated young male mice overexpressing human wild-type $\alpha$-synuclein under control of the Thy-1 promoter (Thy1-aSyn mice) with CLR01 and examined motor
\end{abstract}

Electronic supplementary material The online version of this article (doi:10.1007/s13311-017-0544-9) contains supplementary material, which is available to authorized users.

Marie-Françoise Chesselet

mchesselet@mednet.ucla.edu

Gal Bitan

gbitan@mednet.ucla.edu

1 Department of Neurology, David Geffen School of Medicine, University of California, Los Angeles, Los Angeles, CA, USA

2 Brain Research Institute, University of California, Los Angeles, Los Angeles, CA, USA

3 Institute of Organic Chemistry, University of Duisburg-Essen, Essen, Germany

4 Department of Neurobiology, David Geffen School of Medicine, University of California, Los Angeles, Los Angeles, CA, USA

5 Molecular Biology Institute, University of California, Los Angeles, Los Angeles, CA, USA behavior and $\alpha$-synuclein in the brain. Intracerebroventricular administration of CLR01 for 28 days to the mice improved motor dysfunction in the challenging beam test and caused a significant decrease of buffer-soluble $\alpha$-synuclein in the striatum. Proteinase-K-resistant, insoluble $\alpha$-synuclein deposits remained unchanged in the substantia nigra, whereas levels of diffuse cytoplasmic $\alpha$-synuclein in dopaminergic neurons increased in mice receiving CLR01 compared with vehicle. More moderate improvement of motor deficits was also achieved by subcutaneous administration of CLR01, in 2/5 trials of the challenging beam test and in the pole test, which requires balance and coordination. The data support further development of molecular tweezers as therapeutic agents for synucleinopathies.

Key Words Parkinson's disease - synucleinopathies · mouse model $\cdot$ motor behavior $\cdot \alpha$-synuclein aggregation $\cdot$ drug testing

\section{Introduction}

Synucleinopathies are diseases characterized by cellular accumulation and deposition of $\alpha$-synuclein, a presynaptic protein whose physiological function is not fully understood $[1,2]$. Deposition in intraneuronal Lewy bodies and Lewy neurites is a characteristic of sporadic Parkinson's disease (PD) and dementia with Lewy bodies, whereas glial cytoplasmic inclusions in oligodendrocytes characterize multiple system atrophy [3]. Rare point mutations in SNCA, which encodes $\alpha$ synuclein, or multiplication of the wild-type (WT) gene, cause autosomal dominant familial PD [4]. In addition, multiple genome-wide association studies have identified polymorphisms in SNCA as a major risk factor for developing sporadic PD [5-8]. Additional support for the major role of $\alpha$ - 
synuclein in PD and other synucleinopathies comes from studies of animal models [9-13]. Overexpression of mutant or WT $\alpha$-synuclein in various models causes motor deficits, accompanied by impaired neuronal function and in certain cases, neurodegeneration, reminiscent of human synucleinopathies.

Multiple cellular mechanisms are involved in $\alpha$-synucleininduced neurotoxicity, including oxidative stress, mitochondrial dysfunction, and impaired protein clearance [14, 15]. Similarly to other proteinopathies, the pathology is clearly associated with abnormal self-association of $\alpha$-synuclein monomers into oligomers and insoluble aggregates that can be amorphous or take the form of amyloid fibrils [16]. Which of these forms is a major contributor to the pathologic process is an open question and an area of active investigation. Regardless of the precise answer to this question, strategies for interfering with $\alpha$-synuclein assembly have become the focus of efforts to develop therapy for synucleopathies [17].

The molecular tweezer CLR01 is a novel, broad-spectrum modulator of abnormal self-assembly of multiple amyloidogenic proteins [18] and has a beneficial effect in several animal models of proteinopathies [19, 20]. The mechanism of action of CLR01 is unique. The compound binds exposed Lys residues typically with low micromolar affinity $[18,21,22]$. The binding is highly labile [23], yet it is selective to Lys and involves inclusion of the Lys side-chain within the tweezer cavity [24]. The moderate binding affinity is sufficient for interrupting the weak forces mediating the formation of metastable oligomers and nuclei of amyloidogenic proteins but does not disrupt the structure or function of stable proteins unless substantially higher concentrations are used [19-21, 25].

CLR01 prevents $\alpha$-synuclein fibril formation in vitro at substoichiometric concentration ratios and effectively inhibits the toxicity of both exogenous and endogenous $\alpha$-synuclein in cell culture [26]. The main binding site for CLR01 is at the Nterminal Lys10/12, though additional binding events were observed using mass spectrometry and fluorescence spectroscopy [22]. The strongest binding occurred with $\mathrm{K}_{\mathrm{d}}=0.79 \mu \mathrm{M}$, the lowest $\mathrm{K}_{\mathrm{d}}$ found to date for CLR01 binding to any protein.

In a zebrafish embryo model of $\alpha$-synuclein toxicity, expression of human, WT $\alpha$-synuclein caused severe deformation and most fish died within 48 to $72 \mathrm{~h}$ postfertilization [26]. Addition of 1 to $10 \mu \mathrm{M}$ CLR01 to the water in which the zebrafish embryos developed led to robust rescue of the phenotype and the survival of the developing embryos. Immunohistochemistry (IHC) and Western blot analyses showed that CLR01 prevented formation of $\alpha$-synuclein aggregates in the zebrafish-embryo neurons and by maintaining the protein in a nonaggregated and nontoxic form, allowed rapid degradation of $\alpha$-synuclein, primarily by the proteasome [26]. Similar rescue was observed in a zebrafish embryo model in which dopaminergic neurodegeneration was caused by the PD-linked pesticide Ziram [13], revealing that Ziram- induced toxicity was mediated by endogenous zebrafish synuclein oligomers and/or aggregates [13]. CLR01 also was found to significantly inhibit endogenous synuclein aggregation and ameliorate neuronal death in a lamprey model of spinal-cord injury [27], in which synuclein accumulation had been found to be tightly linked to neurodegeneration [28].

Previous studies have also shown that CLR01 had beneficial therapeutic effects in mouse models of Alzheimer's disease (AD) [29] and familial amyloidotic polyneuropathy [30], and a high safety margin in WT mice [25]. Based on these data, we hypothesized that administration of CLR01 in a mouse model of synucleinopathy might have a beneficial effect and asked whether this effect would associate with reduction of soluble, oligomeric $\alpha$-synuclein, insoluble aggregates of $\alpha$-synuclein, or both. We used mice overexpressing human, WT $\alpha$-synuclein under the murine Thy-1 promoter (Thy 1 aSyn) [31], which has been characterized thoroughly in the Chesselet laboratory. This mouse model reproduces multiple features of PD including motor dysfunction, nonmotor deficits, mitochondrial dysfunction, inflammation, $\alpha$-synuclein pathology, and dopamine loss [15, 32-39]. Our data show that amelioration of motor deficits following treatment with CLR01 associates with clearance of buffer-soluble $\alpha$-synuclein but not insoluble $\alpha$-synuclein aggregates.

\section{Materials and Methods}

\section{CLR01}

The $\mathrm{Na}^{+}$salt of CLR01 was prepared and purified as described previously [21]. For administration to animals, the compound was dissolved in a sterile saline solution.

\section{Animals}

Animal care was provided in accordance with the United States Public Health Service Guide for the Care and Use of Laboratory Animals. All the procedures were approved by the Institutional Animal Care and Use Committee of the University of California, Los Angeles. Two-month-old, male, hemizygous mice overexpressing human, WT $\alpha$-synuclein under the murine Thy-1 promotor (Thy1-aSyn mice) on a mixed hybrid C57Bl/6J $\times \mathrm{DBA} / 2$ (short BDF1) background [32] and WT littermates were used in this study. Transgenic mice and WT littermates were tested in parallel in a randomized sequence by an investigator unaware of the genotype. $\mathrm{N}$ numbers were determined a priori by power analysis as described previously [32] in order to limit the number of animals used to the minimum required for statistical analysis (" $3 \mathrm{R}$ " principles). The genotype was verified by polymerase chain reaction amplification analysis of DNA [31] and reconfirmed at the end of the study for each mouse. Ear tissue in pups 
(punch) and fecal pellets in older animals were used for DNA extraction instead of tail biopsies to reduce stress and pain to the animal for premortem genotyping. Mice were kept on a reverse 12-h light/12-h dark cycle (light off at $11 \mathrm{am}$ ). All testing was performed between 1 and $5 \mathrm{pm}$ during the dark cycle under low light in an allocated room for behavioral experiments adjacent to the animal holding room. Mice were socially housed (except after stereotactic surgery to reduce the risk of damage to the implant) on standard bedding (shredded wood) and material for nest building (Nestlets, Ancare, NY, USA) was provided. Mice had ad libitum access to water and standard rodent diet (NIH-31 modified 7013; Harlan, Envigo, Hayward, CA, USA). Room temperature in the mouse holding room was $23{ }^{\circ} \mathrm{C} \pm 2{ }^{\circ} \mathrm{C}$ and relative humidity was $\sim 60 \%$; values were recorded during the daily animal check.

\section{Drug Administration, Euthanasia, and Tissue Collection}

In some experiments, CLR01 was administered intracerebroventricularly into the left lateral ventricle to WT and Thy1-aSyn mice using osmotic minipumps (Alzet model 1004; Alzet, Cupertino, CA, USA) for 28 days at 0,1 , or $10 \mu \mathrm{M}$ ( $n=16$ per group). The pumps contained $100 \mu$ l solution, which was delivered continuously at $0.11 \mu \mathrm{l} / \mathrm{h}$. Filling, priming, and implantation of the pumps were performed using standard protocols provided by the manufacturer. Briefly, the Alzet brain infusion kit and a stereotaxic apparatus (Kopf, Tujunga, CA, USA) were used. Mice were anesthetized with a combination of pentobarbital $(60 \mathrm{mg} / \mathrm{kg})$ and low concentrations of isoflurane $(0.5-1.5 \%)$. Mice then were positioned in the stereotaxic apparatus. Following preparation of the surgery site with antiseptics, a scalp incision was made midline and the skull exposed. The coordinates for intracerebroventricular infusion into the left lateral ventricle were determined (anterior-posterior $0.4 \mathrm{~mm}$, mediolateral $1.3 \mathrm{~mm}$, dorsoventral $2.5 \mathrm{~mm}$ in relation to bregma according to the stereotaxic atlas of Paxinos and Franklin [40]), and a small hole for insertion of the infusion cannula was drilled into the skull. The cannula was attached to the pump, which was placed subcutaneously at the back of the animal behind the right scapula. Then, the cannula was lowered into the brain and fixed to the skull using a specific adhesive provided with the Alzet brain infusion kit. The wound was sutured and the animal returned to its home cage.

In other experiments, CLR01 was administered subcutaneously to WT and Thy 1 -aSyn mice at 0 or $0.4 \mathrm{mg} / \mathrm{kg}$ per day for 28 days ( $n=16$ per group) using the same pumps, which were implanted on the back behind the right scapula of the animals following the manufacturer's procedure. Drug administration and subsequent data analysis were performed by investigators blinded to treatment and genotype.

On day 28 , the mice were euthanized by intraperitoneal injection of $100 \mathrm{mg} / \mathrm{kg}$ pentobarbital. One half of the mice from each group were perfused transcardially with $4 \%$ paraformaldehyde in phosphate-buffered saline (PBS), the brains were removed and fixed in $4 \%$ paraformaldehyde in PBS overnight, cryoprotected in a PBS-sucrose solution for $48 \mathrm{~h}$, frozen on powdered dry ice, and stored at $-80^{\circ} \mathrm{C}$. The brains of these animals were used for IHC analysis. The other half of the animals was perfused with PBS only and the brains immediately frozen on dry ice. These brains were used for Western blot analysis.

\section{Behavioral Testing}

Balance, coordination, fine motor control, and limb use were measured using the challenging beam and pole tests (both intracerebroventricularly and subcutaneously treated groups), and the cylinder test (only subcutaneously treated groups) following previously published protocols [41] with modifications, as described previously [42-44]. Animals were examined for motor deficits 1 week before the initiation of the treatment (intracerebroventricular only), and at the end of the treatment (both intracerebroventricular and subcutaneous), before they were euthanized. In the challenging beam test and the pole test, the mice were trained for 2 days and recorded on the third day in 5 consecutive trials. Briefly, the beam consists of 4 sections ( $25 \mathrm{~cm}$ each, $1 \mathrm{~m}$ total length) with decreasing width $(3.5-0.5 \mathrm{~cm})$. Animals were trained to traverse the length of the beam from the widest to the narrowest section towards the home cage. On the day of the test a mesh grid was placed over the beam surface and animals were videotaped while traversing the grid-surfaced beam. Videotapes were rated on slow motion by an experimenter blind to genotype and treatment. The number of errors per step (slip through the grid) was measured for each mouse in each trial. For the pole test, animals were placed head upwards on top of a vertical wooden pole, $50 \mathrm{~cm}$ long and $1 \mathrm{~cm}$ in diameter, inside their home cage. On the test day, time to orient downward (turn) and time to descend after the turn were measured in 5 consecutive trials. If a mouse fell off, slid down, or could not complete the task in $30 \mathrm{~s}$, it was defined as a nonperformer and given a cut-off score of $30 \mathrm{~s}$ for time to turn and $30 \mathrm{~s}$ for time to descend. For post-treatment analysis after intracerebroventricular administration, the same protocols were used, but training was not required as mice retain memory from the pretreatment analysis [44]. In the cylinder test, movements of fore- and hindlimbs (number of steps) were measured in a small transparent cylinder placed on a piece of glass with a mirror positioned at an angle underneath [41]. Videotapes were viewed and rated in slow motion by an experimenter blind to genotype and treatment.

\section{Western Blot Analysis}

Analysis was performed by a modification of previously published protocols [15] on animals that were treated 
intracerebroventricularly. Mouse brains were dissected into the 2 hemispheres. The left hemisphere, ipsilateral to the infusion site, was further dissected to isolate the olfactory bulb, motor cortex, frontal cortex, striatum, hippocampus, thalamus, ventral mesencephalon, and cerebellum, and extracted using a 3-step protocol, including: 1) $20 \mathrm{mM}$ Tris $\cdot \mathrm{HCl}$, $150 \mathrm{mM} \mathrm{NaCl}$ (pH 7.4; buffer-soluble); 2) $20 \mathrm{mM}$ Tris $\cdot \mathrm{HCl}$, $150 \mathrm{mM} \mathrm{NaCl}, 0.1 \%$ Triton-X100 (pH 7.4; detergent-soluble); and 3) $5 \mathrm{M}$ guanidine hydrochloride ( $\mathrm{pH} 7.4$; guanidine-soluble), as described previously [45]. The latter fraction was desalted using Zeba spin columns (\#89882; Thermo Scientific, Waltham, MA, USA). Protein concentration was determined by the Bradford assay.

Western blotting was performed either following fractionation by sodium dodecyl sulfate polyacrylamide gel electrophoresis (SDS-PAGE), as described previously [15], or following native PAGE. In SDS-PAGE, proteins were fractionated on $10 \%$ Bis-Tris gels (Invitrogen, NuPAGE Novex \#NP0303BOX; Carlsbad, CA, USA) using $1 \times X$ SDS running buffer (Invitrogen NuPAGE MOPS; \#NP0001) and transferred to nitrocellulose membranes using an Invitrogen X-cell II blot module apparatus.

In native PAGE experiments, proteins were fractionated on $4 \%$ to $16 \%$ NativePAGE Bis-Tris gels (\#BN1004BOX; Thermo Scientific) using $1 \times$ native PAGE running buffer (\#BN2001; Thermo Scientific) on the outer anode chamber and dark-blue cathode buffer (\#BN2002; Thermo Scientific) to one-third of the run and on the inner cathode chamber at $140 \mathrm{~V}$ for $90 \mathrm{~min}$. The proteins were transferred to polyvinylidene fluoride membranes, incubated in $8 \%$ acetic acid for $15 \mathrm{~min}$ to fix the proteins and reactivated in methanol.

Both SDS-PAGE nitrocellulose membranes and native PAGE polyvinylidene fluoride membranes were blocked (StartingBlock T20, \#37539; Thermo Scientific) for $1 \mathrm{~h}$ at room temperature. Then, membranes were incubated with mouse anti- $\alpha$-synuclein monoclonal antibody (\#610787; BD Transduction Laboratories, BD Biosciences, San Jose, CA, USA) at a $1: 50,000$ dilution or rabbit anti- $\beta$-actin polyclonal antibody (\#ab75186; Abcam, Cambridge, UK) at a 1:5000 dilution in blocking buffer overnight at $4{ }^{\circ} \mathrm{C}$. Then, the membranes were washed with $0.2 \%$ Tween- 20 in 0.1 M PBS and incubated with horseradish peroxidase-conjugated goat antimouse polyclonal antibody (Millipore, Billerica, MA, USA) at a 1:20,000 dilution or goat antirabbit polyclonal antibody (Millipore) at a 1:10,000 dilution. The blots were developed using SuperSignal West Dura (Thermo Scientific) and exposed to Kodak biomax film (Sigma, St. Louis, MO, USA). Bands were scanned and quantified using Image J [46]. In native PAGE, proteins do not separate according to size; therefore, recombinant $\alpha$-synuclein cross-linked using photoinduced cross-linking of unmodified proteins [47] was used as oligomer size reference. The optical density (OD) values of $\alpha$ synuclein bands were normalized to $\beta$-actin as a loading control and graphically presented as OD (SDS-PAGE) or percentage OD (native PAGE) after statistical analysis of absolute $O D$ values.

\section{IHC and Immunofluorescence}

Coronal sections of $40 \mu \mathrm{M}$ were cut on a Zeiss cryostat and used for IHC analysis of $\alpha$-synuclein expression in tyrosine hydroxylase (TH) neurons or of $\alpha$-synuclein aggregation, following pretreatment with proteinase $\mathrm{K}$, in the substantia nigra ( $\mathrm{SN} ;-3.15 \mathrm{~mm}$ in relation to bregma according to the stereotaxic atlas of Paxinos and Franklin [40]) using an antibody that recognizes both mouse and human $\alpha$-synuclein (mouse anti- $\alpha$-synuclein monoclonal antibody, BD Transduction Laboratories, clone 42, \#610787; BD Biosciences) at a 1:500 dilution, as described previously $[42,48,49]$. Sections for each staining protocol were processed in a single experiment. Briefly, for $\alpha$-synuclein and TH co-labeling of nigral sections (Rabbit anti-TH, diluted 1:600; Millipore), secondary antibodies were conjugated with Alexa 488 ( $\alpha$-synuclein) or Alexa 594 (TH). To measure $\alpha$-synuclein staining in dopaminergic nigrostriatal neurons, immunofluorescence doublelabeled images from the SN were acquired at $40 \times$ under constant imaging conditions using a Leica TCS SP5 MP-STED microscope. Fluorescence intensity for $\alpha$-synuclein immunoreactivity was measured in all TH-positive neurons of the SN that were in focus in one section per animal using ImageJ as described previously [42].

Quantitative analysis of proteinase K-resistant $\alpha$-synuclein aggregate number, surface occupied by aggregates, and aggregate size distribution was performed in the SN. One SN section pretreated with proteinase $\mathrm{K}$ and immunostained for $\alpha$ synuclein was used per transgenic mouse (WT mice do not develop proteinase K-resistant aggregates of $\alpha$-synuclein and therefore were not analyzed [49]). The contour of the SN was delineated at $5 \times$ objective using the StereoInvestigator software coupled to a Leica DM-LB microscope with a Ludl XYZ motorized stage. Four images across the SN then were acquired and subjected to particle analysis in ImageJ as described previously [42]. The surface area covered by aggregates was measured and the number of aggregates was calculated per $100 \mu^{2}$.

\section{Blood-Brain Barrier Penetration}

Two-month-old, male WT or Thy1-aSyn mice were used $(n=$ 3 per time point). Mice were administered ${ }^{3} \mathrm{H}$-CLR01 intravenously as described previously [25]. In previous experiments, the background signal of naïve mouse brain equaled the signal of the environmental background; therefore, no additional control group for background subtraction was included. Briefly, a mixture of $90 \%$ CLR01 and $10 \%{ }^{3} \mathrm{H}$-CLR01 was injected at $2 \mu \mathrm{Ci}$ and $11.86 \mu \mathrm{g}$ total CLR01 per $\mathrm{g}$ of 
mouse body weight. Blood and brain were collected at 1, 3, or $8 \mathrm{~h}$ following administration of CLR01. One hemisphere of the brain and 100 to $350 \mu$ of blood were collected and separately digested. Digested tissue was added to Ultima Gold Liquid Scintillation Cocktail (Perkin-Elmer, Waltham, MA, USA) and read in a Triathler Liquid Scintillation Counter model 425-034, (Hidex, Turku, Finland). CLR01 brain penetration was calculated as counts per minute (CPM) per g of brain relative to CPM per $\mathrm{ml}$ of blood.

\section{Statistics}

Analysis of variance (ANOVA) was used as a multicomparison procedure followed by Bonferroni's posthoc test for parametric data. Mann-Whitney $U$ and Wilcoxon signed rank tests were used for nonparametric comparisons. The null hypothesis was rejected at a $p$-value $<0.05$. Distribution of fluorescence intensity of $\alpha$-synuclein in THpositive neurons does not follow a Gaussian distribution; therefore, we applied a bootstrapping method using custom MATLAB functions as done previously $[42,50]$. The comparison was considered to be significant at the $p<0.05$ level when the sample parameter fell outside of the $95 \%$ confidence interval of the control group, independent of assumptions about the probability distribution.

\section{Results}

In a first set of experiments, to maximize exposure of the relevant brain regions to CLR01, the compound was administered intracerebroventricularly and the left hemisphere, ipsilateral to the infusion site, was analyzed for $\alpha$-synuclein. The CLR01 concentrations used in these experiments, 1 or $10 \mu \mathrm{M}$ in osmotic pumps, were rough estimates based on the concentrations used in previous zebrafish experiments $[13,26]$.

\section{Intracerebroventricular Administration of CLR01 for 28 Days is Safe}

The safety of peripheral administration of CLR01 in mice was demonstrated in previous studies [25, 29, 30], yet the current study was the first to administer CLR01 directly into the lateral ventricle of mice. Therefore, it was important to assess the safety of the treatment. We found that administration of CLR01 did not cause any signs of overt toxicity or reduction in general health status and did not affect body weight for the duration of the treatment. As described previously, Thy 1-aSyn mice at this age showed slightly lower body weights than WT mice [32]. Nonetheless, there was no significant difference in weekly body weight measurements between the groups treated with vehicle and those treated with CLR01 (Fig. S1).
Intracerebroventricular Administration of CLR01 Improves Motor Behavior in Thy1-aSyn Mice

The mice were administered CLR 01 or vehicle between 2 and 3 months of age because behavioral deficits in the Thy1-aSyn mouse model are detected already at about 1 to 2 months of age and worsen progressively $[32,51]$. The effect of CLR01 on the progression of motor deficits was examined by comparing motor performance prior to drug administration, at $\sim 2$ months of age ( 1 week before pump implantation) and 5 weeks later, at the end of treatment, at about 3 months of age (on day 28 postpump implantation).

At 2.5 months of age, Thy 1-aSyn mice make significantly more foot slips through the meshed grid (errors per step) in the challenging beam test than WT mice. As expected, vehicletreated Thy 1-aSyn mice showed a progressive increase in deficit (errors per step) in the challenging-beam test between 2 and 3 months of age, from $0.43 \pm 0.03$ to $0.53 \pm 0.03$ [2-way repeated-measures ANOVA for vehicle-treated groups, genotype $\times$ time-point interaction $\mathrm{F}(1,27)=11.8, p=0.002$; Bonferroni $t$ test: Thy 1 -aSyn $v s$ WT at pre- or post-treatment $p<0.001$; Thy 1-aSyn pre- $v s$ post-treatment $p<0.001$, WT pre- $v s$ post-treatment nonsignificant (NS)] (Fig. 1a). CLR01 halted the progression of the deficit in mice receiving either low or high doses of CLR01 (2-way repeated measures ANOVA for 1 and $10 \mu \mathrm{M}$, factor time point NS) (Fig. 1a). Furthermore, at $1 \mu \mathrm{M}$, CLR01 led to a significant and robust reduction in errors per steps in the Thy1-aSyn mice compared with vehicle-treated mice, both when analyzed as a mean of 5 trials [post-treatment, 2-way ANOVA genotype $\times$ treatment interaction $\mathrm{F}(2,78)=4.01, p=0.022$; Bonferroni $t$ test: Thy1-aSyn vehicle $v s$ CLR01 $1 \mu \mathrm{M} p=0.002$ ] (Fig. 1a) and for each single trial (Fig. 1b). In the first trial the improvement reached $39.3 \%$ compared with vehicle treated-Thy1-aSyn mice, whereas the average improvement in the 5 trials was $29.2 \%$. At $10 \mu \mathrm{M}$, the improvement was less pronounced and the deficits were reduced only in the first and third trials (Fig. 1c), suggesting hormesis.

In contrast to the marked effect in the challenging beam test, there was no improvement in the pole test after intracerebroventricular administration of CLR01 (Fig. S2). Possible reasons for this marked difference between the challenging beam and pole tests following intracerebroventricular administration of CLR01 may be attributed to distribution of the compound in the brain or to peripheral effects (see below and "Discussion").

\section{CLR01 Decreases Buffer-Soluble, But Not Insoluble Striatal $\alpha$-Synuclein}

Thy1-aSyn mice overexpress human WT $\alpha$-synuclein starting at 7 to 10 days after birth [32]. The ventral mesencephalon containing the $\mathrm{SN}$ and the striatum of the left hemisphere, 


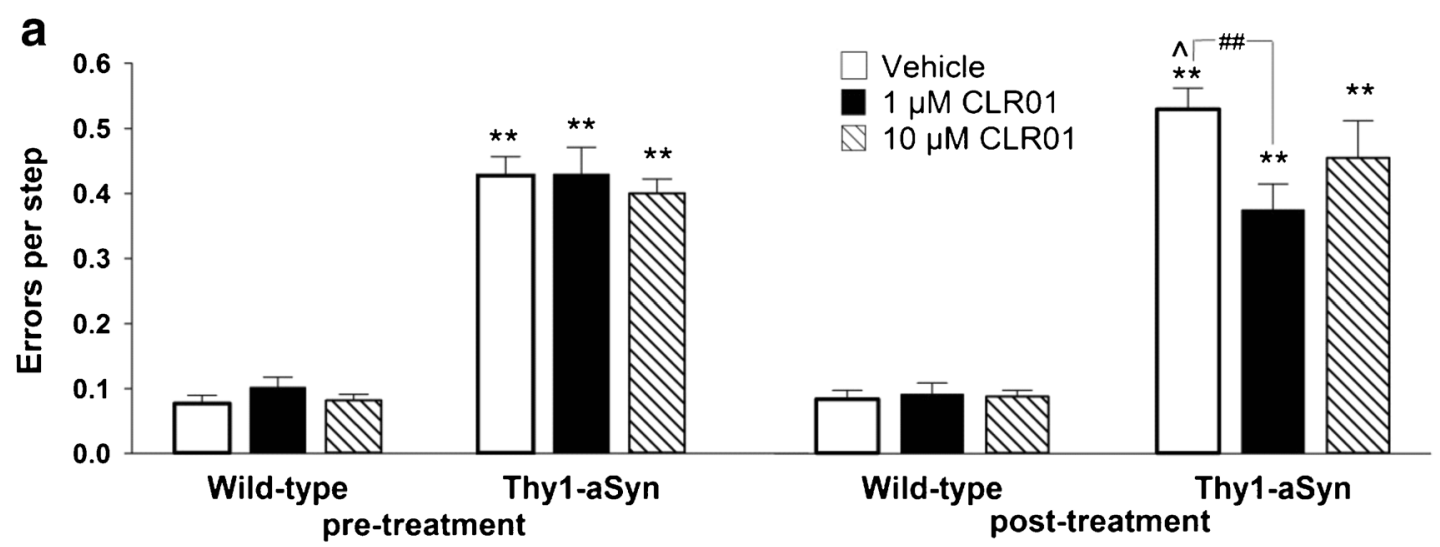

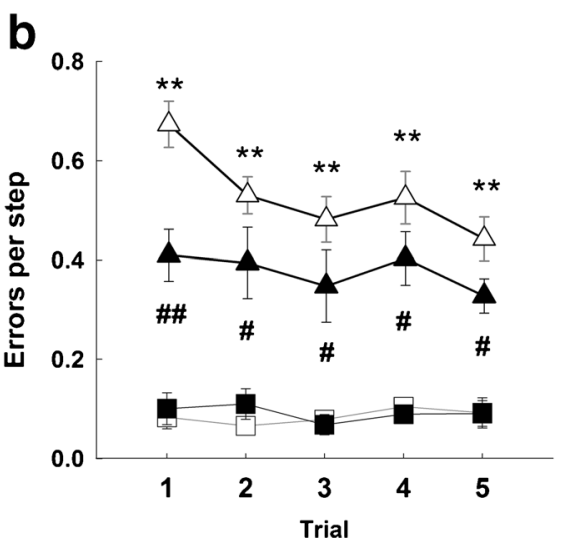

Fig. 1 Intracerebroventricular CLR01 administration improves performance in the challenging-beam test. (a) Mean + SEM errors per step on the challenging-beam before (pre-) and after (post-) treatment $\left[{ }^{* *} p<0.01 v s\right.$ the respective wild-type (WT) groups; ${ }^{\# \#} p<0.01 v s$ the vehicle-treated and $1-\mu \mathrm{M}$-treated groups; $\hat{p}<0.01$ vs vehicle-treated group before and after treatment, 2-way analysis of variance (ANOVA)]. (b) Errors per step (mean \pm SEM) in individual trials for mice

ipsilateral to the infusion site, were dissected for examination of the effects of CLR01 treatment on $\alpha$-synuclein concentration levels. The tissue was extracted using a 3-step protocol, fractionating the proteins into a buffer-soluble fraction containing cytoplasmic and water-soluble extracellular proteins, detergent-soluble membrane-associated proteins, and insoluble proteins from inclusion bodies and aggregates, which were extracted using $5 \mathrm{M}$ guanidine $\mathrm{HCl}$ (guanidine-soluble). Total $\alpha$-synuclein in each fraction was quantified by densitometry of Western blots using an antibody that recognizes both mouse and human $\alpha$-synuclein.

In all the fractions of the striatum and ventral mesencephalon extracts, significant accumulation of $\alpha$-synuclein was found in the Thy1-aSyn mice, as expected (Fig. 2). CLR01 administration led to a significant reduction in buffer-soluble $\alpha$-synuclein in the striatum of the Thy1-aSyn mice at both $1 \mu \mathrm{M}(42 \pm 12 \%)$ and $10 \mu \mathrm{M}(39 \pm 13 \%)$ [2-way ANOVA, genotype $\times$ treatment interaction $\mathrm{F}(2,42)=4.33, p=0.02$; Bonferroni $t$ test: Thy1-aSyn vehicle $v s 1 \mu \mathrm{M}$ or $10 \mu \mathrm{M}$
CLR01 $p<0.05$; WT vehicle $v s 1 \mu \mathrm{M}$ or $10 \mu \mathrm{M}$ CLR01 NS] (Fig. 2a, c). A smaller and highly variable, nonsignificant reduction $(25 \pm 22 \%)$ was observed in the $10 \mu \mathrm{M}$ group in the ventral mesencephalon (Fig. 2b, d). In the detergent-soluble

Fig. 2 The effect of CLR01 on brain $\alpha$-synuclein. Brains from wild-type (WT) and Thy 1-aSyn mice were extracted into buffer-soluble, detergentsoluble, and guanidine-soluble fractions. Each fraction was analyzed by Western blotting with an anti- $\alpha$-synuclein antibody and bands corresponding to $\alpha$-synuclein were quantified densitometrically and normalized to $\beta$-actin, which was used as a loading control. $(\mathrm{a}, \mathrm{b})$ Representative blots of buffer-soluble and detergent-soluble fractions. (c-h) Quantification of normalized $\alpha$-synuclein. The data in panels $(\mathrm{c}-\mathrm{h})$ are presented as mean + SEM. Grubb's test was performed to remove outliers and 1 outlier was removed from the striatal detergent-soluble fraction of Thy1-aSyn/1 $\mu \mathrm{M}(n=6)$ group. The difference between the WT and transgenic mice was statistically significant $(p<0.001)$ in all cases (\#p $<0.05$ vs vehicle-treated Thy1-aSyn mice, 2-way ANOVA). $n_{\text {(WT }}$ vehicle $)=5, n_{(\mathrm{WT} / 1 \mu \mathrm{M})}=3, n_{(\mathrm{WT} / 10 \mu \mathrm{M})}=7, n_{(\text {Thy } 1 \text {-aSyn } / \text { vehicle })}=6, n_{(\text {Thy } 1-}$ $\mathrm{aSyn} / 1 \mu \mathrm{M})=6, n_{(\text {Thyl }-\mathrm{aSyn} / 10 \mu \mathrm{M})}=5$ 


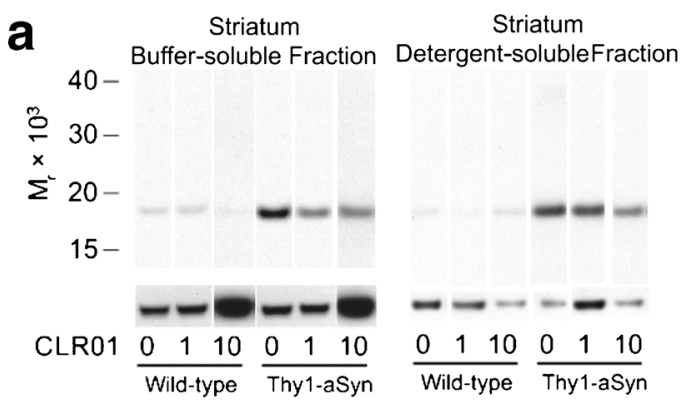

Ventral Mesencephalon Ventral Mesencephalon
Buffer-soluble Fraction Detergent-solubleFraction
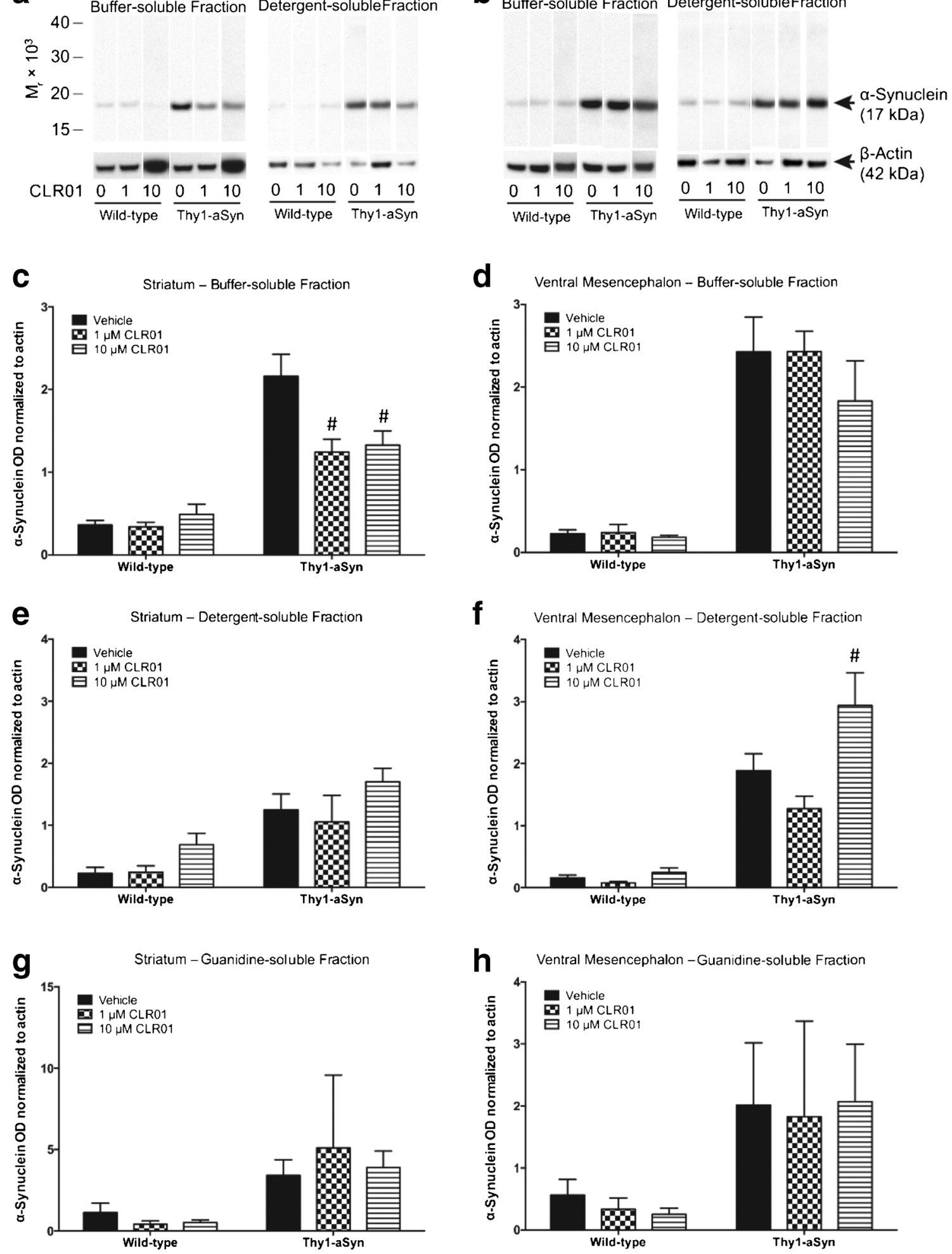

fraction, there was a significant increase $(56 \pm 16 \%)$ in $\alpha$ synuclein in the high-dose group in the ventral mesencephalon but not in the low-dose group [2-way ANOVA, genotype $\times$ treatment interaction $\mathrm{F}(2,42)=4.22, p=0.02$; Bonferroni $t$ test: Thy1-aSyn vehicle vs $10 \mu \mathrm{M}$ CLR01 $p<0.05$, vehicle

vs $1 \mu \mathrm{M}$ CLR01 NS; WT vehicle $v s 1 \mu \mathrm{M}$ or $10 \mu \mathrm{M}$ CLR01 NS] (Fig. 2b, f), or in the striatum (Fig. 2a, e). There were no significant differences in the guanidine-soluble fraction among the treatment groups in either the ventral mesencephalon or the striatum (Fig. $2 \mathrm{~g}, \mathrm{~h}$ ). 
The ventral mesencephalon block used for Western blot analyses contains the cell bodies of the dopaminergic neurons located in the SN but also other cells and regions. To further assess the effects of CLR01 on $\alpha$-synuclein specifically in the $\mathrm{SN}$, we performed IHC experiments with the same anti- $\alpha$ synuclein antibody used for the Western blots. In agreement with previous reports [42, 49], numerous proteinase-Kresistant $\alpha$-synuclein aggregates were detected in the $\mathrm{SN}$ of Thy1-aSyn mice (Fig. S3). Concurring with the absence of treatment effect on the guanidine-soluble $\alpha$-synuclein fraction (Fig. $2 \mathrm{~g}, \mathrm{~h}$ ), there were no changes in proteinase-K-resistant $\alpha$-synuclein aggregates in either of the CLR01-treated groups relative to the vehicle group (Fig. S3).

To gain further level of detail, we used confocal microscopy to assess the levels of $\alpha$-synuclein immunostaining in the cytoplasm of dopaminergic neurons in the SN of Thy1-aSyn mice, which were identified by the presence of immunostaining for TH. We observed a significant increase in the number of TH-positive cells that expressed high levels of $\alpha$-synuclein in the left SN ipsilateral to the infusion site in Thy1-aSyn mice administered $1 \mu \mathrm{M}$ and, to a lesser extent, $10 \mu \mathrm{M}$ CLR01 (Fig. 3a, b).
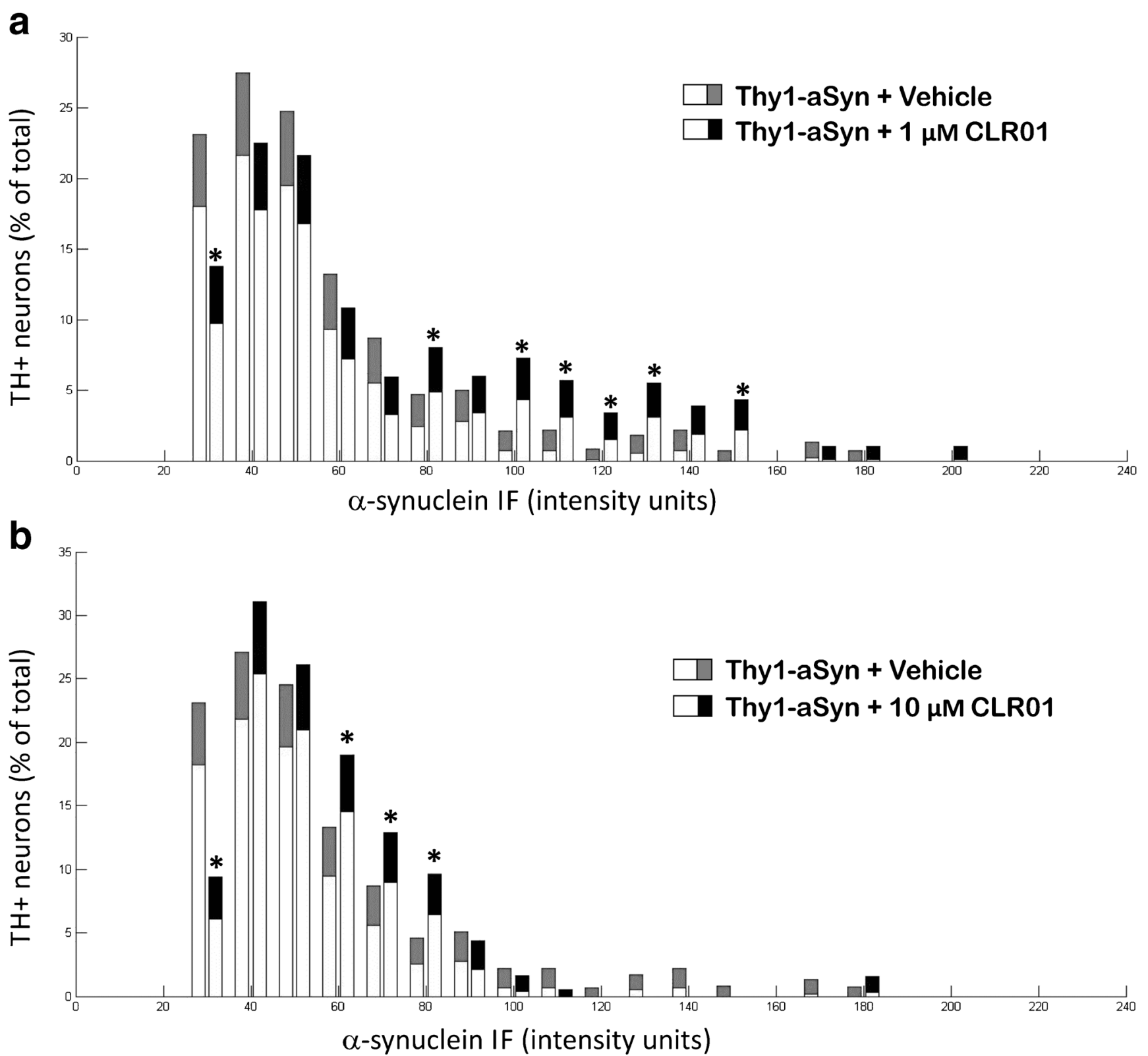

Fig. 3 CLR01 effects on $\alpha$-synuclein in tyrosine hydroxylase (TH) neurons of the substantia nigra (SN). (a) $1-\mu \mathrm{M}$ and (b) 10- $\mathrm{MM}$ CLR01 decreased the percentage of neurons in the left $\mathrm{SN}$, ipsilateral to the infusion site, with low $\alpha$-synuclein immunofluorescent (IF) intensity while increasing the percentage of neurons with high $\alpha$-synuclein IF intensity (bootstrapping analysis displayed as means $+95 \%$ confidence intervals, $* p<0.05, n=5$ per group) 
Because the group administered $1 \mu \mathrm{M}$ CLR01 showed both a significant improvement in the challenging-beam test and a reduction of buffer-soluble $\alpha$-synuclein in the striatum, we asked whether specific $\alpha$-synuclein oligomers could be identified, which were significantly modulated by CLR01. To address this question, we analyzed the buffer-soluble fraction from the vehicle-treated and $1 \mu \mathrm{M}$ CLR01-treated groups using native PAGE. The analysis showed 3 main bands, corresponding to a monomer, a band with an apparent mobility between dimer and trimer compared with recombinant $\alpha$-synuclein cross-linked using photo-induced cross-linking of unmodified proteins [47], and a high-molecular-weight "smear" (Fig. S4a). In comparison, the WT mice showed very little $\alpha$-synuclein. The variations in abundance of the 2 lower-molecular-weight bands among all Thy1aSyn mice were small, whereas the abundance of the high-molecular-weight band varied substantially (Fig. S4a). However, there was no significant difference in the abundance of any of the bands among the different treatment groups in the ventral mesencephalon (Fig. S4b) or the striatum (Fig. S4c).
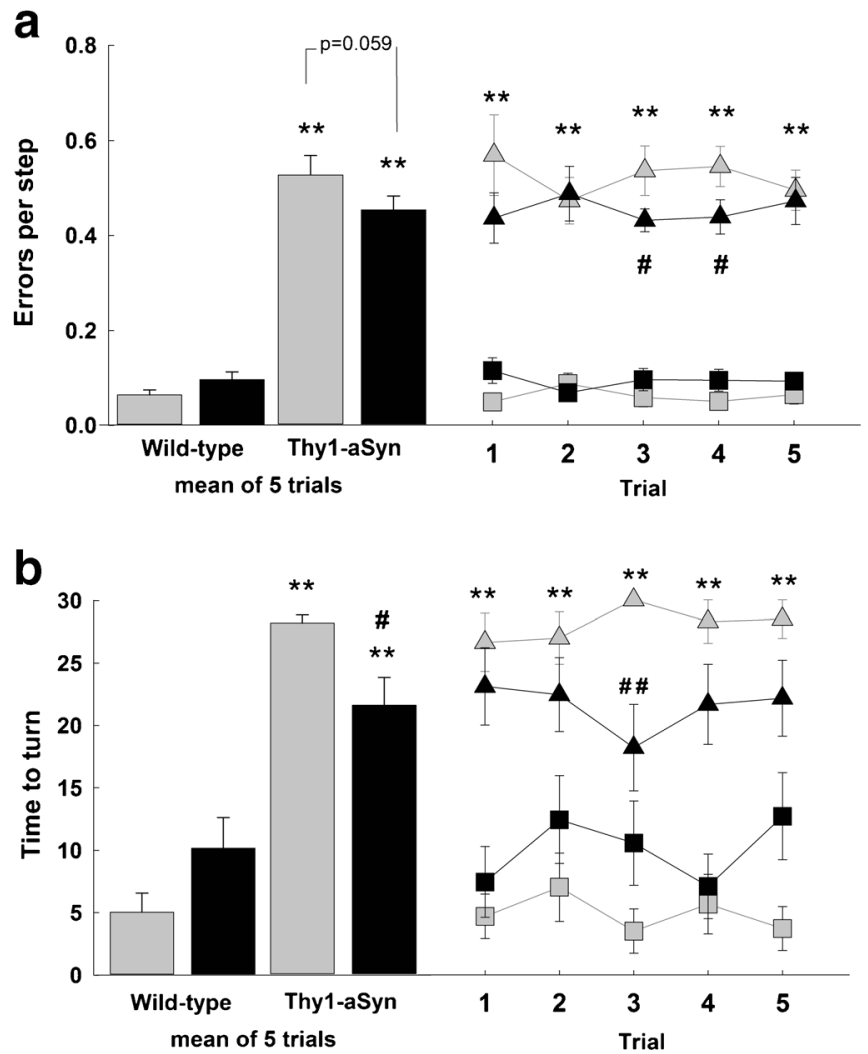

Fig. 4 CLR01 improves motor deficits following subcutaneous administration. (a) Mean \pm SEM of 5 trials and single trials of errors per step on the challenging beam test $[* * p<0.01 v s$ the respective wild-type (WT) mice; ${ }^{\#} p<0.05$ vs the vehicle-treated mice, 2 -way analysis of variance genotype $\times$ treatment for each trial, Bonferroni $t$ test, $n=16$ /group].

\section{Systemic Administration of CLR01 Improves Motor Deficits}

Following the demonstration that direct central CLR01 administration improved motor deficits in the Thy1-aSyn mice, we asked whether peripheral administration also could improve the disease phenotype. To answer this question, mice were administered CLR01 or vehicle at $0.4 \mathrm{mg} / \mathrm{kg}$ per day subcutaneously via osmotic minipumps for 28 days and were examined using the pole, beam, and cylinder tests during the final 3 days of administration.

CLR01-treated mice showed a trend $[14 \%, p=0.059$, 2way ANOVA genotype $\times$ treatment, genotype $\mathrm{F}(1,60)=229$, $p<0.001$; treatment $p>0.05$; interaction $p=0.054$; Bonferroni $t$ test] towards reduced errors per step analyzed as a mean of 5 trials, and a significant reduction in errors per step on trials 3 [19\%, 2-way ANOVA genotype $\times$ treatment interaction $\mathrm{F}(1,60)=4.8, p<0.03$; Bonferroni $t$ test $p<0.05]$ and 4 [19\%, 2-way ANOVA genotype $\times$ treatment interaction $\mathrm{F}(1,60)=5.9, p<0.018$; Bonferroni $t$ test $p<0.05]$ out of 5 trials (Fig. 4a). Furthermore, subcutaneous administration of CLR01 reduced the deficit in the pole test, resulting in a $24 \%$
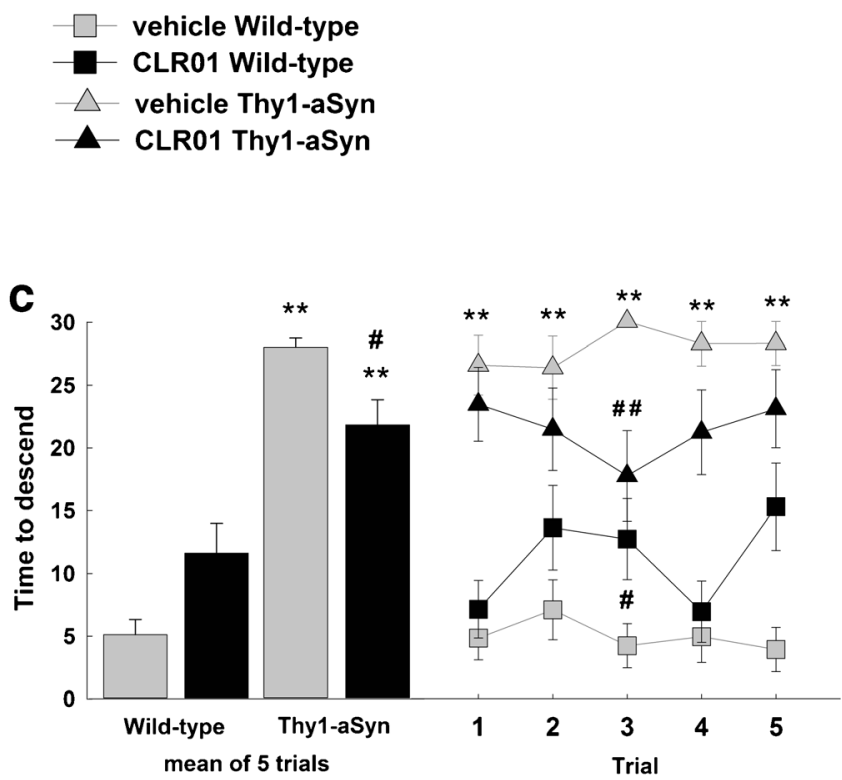

(b) Time to turn in the pole test (mean and single trials) and (c) time to descend in the pole test (mean and single trials) $(* * p<0.01 v$ s the respective WT mice; ${ }^{\#} p<0.05$ and ${ }^{\# \#} p<0.01$ vs the vehicle-treated mice, Mann-Whitney $U$ test, $n=16$ /group) 
improvement in time to turn (Fig. 4b) and $22 \%$ improvement in time to descend (Fig. 4c). Mice take roughly similar time to turn and to descend after the turn, and both measures were similarly improved by CLR01 (Fig. 4b, c). Similar to the effect in the challenging beam test, the reduction was most pronounced in trial 3 (40\%, $p<0.05$ Mann-Whitney $U$ test) out of 5 trials (Fig. 4b, c). The number of mice able to perform the task within $60 \mathrm{~s}$ (cut off time to turn plus time to descend) increased in Thy1-aSyn mice receiving CLR01 (performers: 6/16 vehicle-treated mice vs 11/16 CLR01-treated mice), but this difference was not statistically significant $(p=0.156$ Fisher's exact test). These improvements of motor performance were not related to changes in limb use: CLR01 treatment did not affect reduction of hindlimb use in Thy1-aSyn mice compared with WT mice when turning in a small cylinder (Fig. S5).

\section{Blood Brain Barrier Permeability of CLR01 is Similar in Thy1-aSyn and WT Mice}

The smaller improvement of mice administered CLR01 subcutaneously in the challenging beam test compared with the effect following intracerebroventricular administration raised the question of CLR01's ability to penetrate the blood-brain barrier (BBB) of the Thy1-aSyn mice. To address this question, we used ${ }^{3} \mathrm{H}$-labeled CLR01, as described previously [25], to measure BBB penetration. CLR01 spiked with ${ }^{3} \mathrm{H}-$ CLR01 was administered intravenously to WT or Thy1-aSyn mice. To correct for the radioactivity associated with bloodborne ${ }^{3} \mathrm{H}$-CLR01 in the brain vasculature, we calculated radioactivity associated with $10 \mu$ l blood per $g$ of brain and subtracted this value from the brain radioactivity levels. This method is a common practice in BBB-permeability studies
$[52,53]$ and removes variability among mice associated with a perfusion step where liver color is used as an indirect readout of brain perfusion levels [25].

One $\mathrm{h}$ after injection of ${ }^{3} \mathrm{H}$-CLR01, brain radioactivity levels, calculated as a percentage of blood radioactivity levels $[(\mathrm{CPM} / \mathrm{g}) /(\mathrm{CPM} / \mathrm{ml}) \times 100]$ were indistinguishable between the WT and Thyl-aSyn mice (WT: $2.8 \pm 1.3 \%$; Thy1-aSyn: $2.7 \pm 2.0 \%$ ). These values were also comparable with those observed in the $3 \times \mathrm{Tg} \mathrm{AD}$ mice [25], suggesting that the BBB penetration level of CLR01 is independent of disease mechanism. Similarly to the previous report [25], though blood levels of CLR01 declined with $\mathrm{t}_{1 / 2} \approx 2$ to $3 \mathrm{~h}$, the brain levels remained steady up to $8 \mathrm{~h}$, the latest time point measured (Fig. 5), suggesting that clearance from the brain occurs with substantially slower kinetics than clearance from the blood. For clarity, the data are presented in terms of total radioactivity $(\mathrm{CPM} / \mathrm{g})$ rather than $\mathrm{BBB}$ penetration. $\mathrm{BBB}$ penetration would have appeared to increase owing to the decline in blood levels, while brain levels remain steady. The data suggest that upon continuous administration of CLR01 over 28 days, the compound likely accumulates in the brain up to a steady-state, providing a plausible explanation for the observed effects on motor deficits, despite the initial low BBB penetration levels.

\section{Discussion}

Counteracting $\alpha$-synuclein toxicity is an attractive target for development of disease-modifying treatment for PD and other synucleinopathies, all of which are cureless and highly debilitating diseases. For treatments targeting $\alpha$-synuclein to be effective, knowledge of the most relevant abnormal assemblies of the
Fig. 5 CLR01 persists in the brain $8 \mathrm{~h}$ postadministration. Wild-type or Thy1-aSyn mice were administered $2 \mu \mathrm{Ci}$ of ${ }^{3} \mathrm{H}$ CLR01 spiked into unlabeled CLR01 and the radioactivity in the brain was measured at 1,3 , and $8 \mathrm{~h}$ ( $n=3$ mice per time point) using scintillation counting $($ mean + SEM)

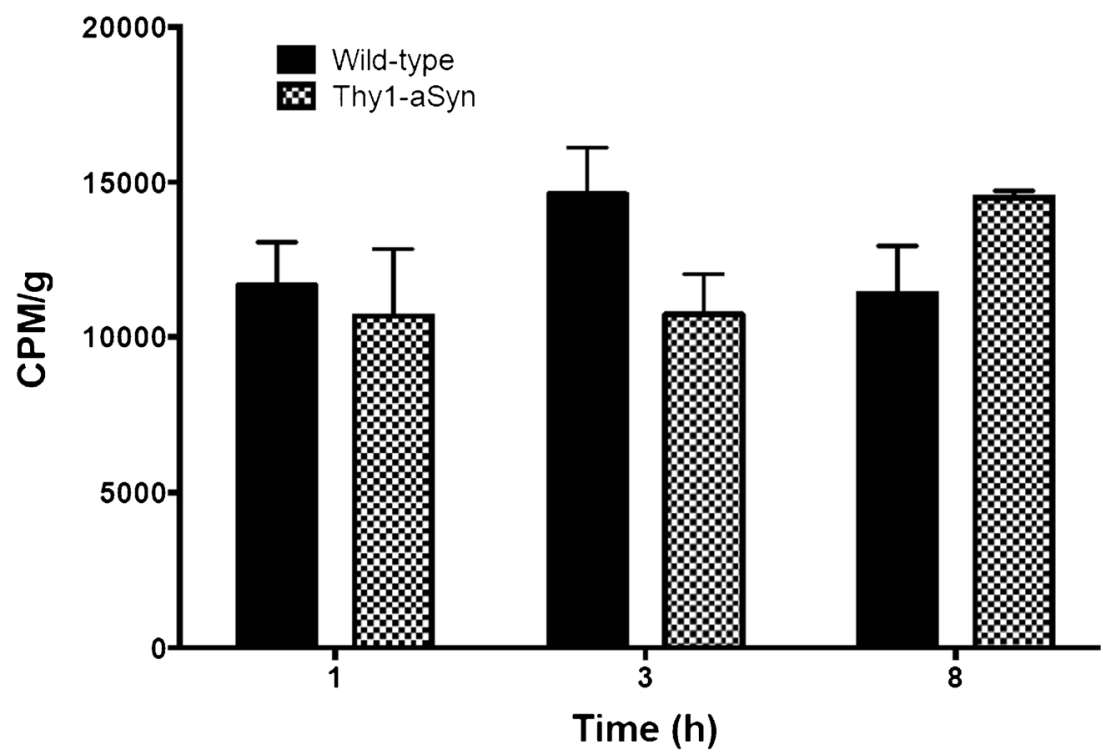


protein is necessary. Our data demonstrate that reduction of buffer-soluble $\alpha$-synuclein in the striatum in mice overexpressing the human, WT form of the protein (Fig. 2a, c) associates with improvement of motor deficits (Fig. 1a, b), without requiring change in aggregated $\alpha$-synuclein deposits in the SN (Fig. S3). CLR01 binds noncovalently to $\alpha$-synuclein and modifies its assembly. By this mechanism, the compound reduces pathologically accumulated $\alpha$-synuclein oligomers by remodeling the protein into nontoxic, nonfibrillar assemblies that are amenable to clearance $[21,26,27]$. The association between up to $39 \%$ improvement in the challenging-beam test after intracerebroventricular infusion and a reduction in buffer-soluble, low-molecularweight $\alpha$-synuclein in the striatum supports the notion that soluble oligomeric forms of $\alpha$-synuclein may be the predominant effectors of neuronal dysfunction.

Notably, functional benefit was achieved without reversal of insoluble aggregate formation. The lack of reduction in $\alpha$ synuclein aggregates (Fig. S3) contrasts with the decrease in amyloid plaques and neurofibrillary tangles observed in a previous study in an AD mouse model [29]. Whereas in the $3 \times$ Tg mouse model of $A D$, deposition of $A \beta$ begins at $\sim 9$ months of age and tau aggregation occurs even later [54], abundant $\alpha$ synuclein aggregation and deposition is observed in the Thy1aSyn mouse by 2 months of age [32, 49]. As CLR01 was administered in a similar manner in both studies and its BBB penetration is similar in both models ([25]; Fig. 5), the concentration of the compound in the brain might have been sufficient for inducing clearance of buffer-soluble $\alpha$-synuclein, but not of insoluble $\alpha$-synuclein aggregates (guanidinesoluble fraction). In support of this hypothesis, in vitro equimolar concentration of CLR01 is sufficient for efficient inhibition of $\alpha$-synuclein fibril formation, whereas a 10 -fold excess CLR01 is required for slow dissociation of mature $\alpha$ synuclein fibrils over 2 months [26]. Our data argue that dissociation of $\alpha$-synuclein fibrils may not be required for functional improvement. Notably, in a study in which $\alpha$-synuclein expression was reduced via antisense oligonucleotide intracerebroventricular infusion in Thy1-aSyn mice, we observed substantial reduction in both the buffer-soluble and insoluble $\alpha$-synuclein aggregates in the SN. However, no further improvement was observed in the functional benefit in the challenging beam test, and the results were highly similar to the effects of CLR01 [55]. These observations support the hypothesis that the crucial factor for the functional improvement was the decrease in buffer-soluble $\alpha$-synuclein in the striatum, rather than in nigral $\alpha$-synuclein aggregates. This interpretation is also consistent with extensive evidence in models of proteinopathies, such as Huntington's disease, that deposition of aggregated proteins in neurons may not correlate with neuronal dysfunction $[56,57]$. Similarly, genetic experiments have related behavioral deficits to the formation of $\alpha$ synuclein oligomers but not fibrillar aggregates [58]. At the young age used in the current study, Thy1-aSyn mice already show alterations in corticostriatal circuitry $[32,39]$ and it is conceivable that reduction of pathological $\alpha$-synuclein by CLR01 improved synaptic function in the striatum. These results also agree with a recent report of the effect of CLR01 in a lamprey model of spinal cord injury [27].

Which exact species of $\alpha$-synuclein oligomers were reduced by CLR01 is difficult to define, as detecting specific $\alpha$-synuclein oligomers in vivo and correlating between oligomer size and toxic activity is a major challenge in PD and other proteinopathies [59]. Native PAGE has been used previously to assess the effect of CLR01 on $\alpha$-synuclein oligomerization in vitro [26]. Here, we used this method to assess the buffersoluble fraction from mice treated intracerebroventricularly with CLR01 or vehicle. Though the method distinguished monomers, small oligomers, and large oligomers, there was no significant difference between the treatment groups analyzed (Fig. S4), even though overall the buffer-soluble fraction showed a robust, significant reduction in $\alpha$-synuclein following CLR01 treatment in SDS-PAGE (Fig. 2a, c). As CLR01 only affects the selfassembly but not the expression of $\alpha$-synuclein [21, 27], it is still plausible that reduction in oligomeric $\alpha$-synuclein was responsible for the decrease of $\alpha$-synuclein load in the striatum. These native PAGE results reflect the previously observed difficulty to detect accurately changes in $\alpha$-synuclein oligomerization in vivo. The low resolution of native PAGE might have contributed to the insufficient sensitivity of the method.

Despite much effort to develop antibodies specific for pathologic forms of $\alpha$-synuclein, there is no perfect method to identify different $\alpha$-synuclein forms by IHC, the only method that provides the anatomical resolution needed to examine specifically nigrostriatal dopaminergic neurons. Using a confocal microscopy method that previously allowed us to document changes of $\alpha$-synuclein levels after drug treatment [42], we observed increases in $\alpha$-synuclein immunostaining after CLR01 administration in TH-positive neurons of the SN in Thy1-aSyn mice. The specific increase in detergent-soluble $\alpha$ synuclein detected via SDS-PAGE in the ventral mesencephalon of the high-dose treatment group suggests that membranebound $\alpha$-synuclein could be increased by CLR01 in this region. Such an increase may explain the increase in immunofluorescent signal seen in dopaminergic cell bodies at lower concentration of CLR01 than with Western blots, which included a wide range of cells, which potentially diluted the effect.

The addition of the detergent in SDS-PAGE limits quantification to total $\alpha$-synuclein as it precludes assessment of the assembly state of the protein, which is likely altered by the presence of detergent [60]. The lack of effects on buffer-soluble $\alpha$-synuclein in the ventral mesencephalon, however, argues against unspecific $\alpha$-synuclein accumulation or lack of clearance in this region after CLR01 administration. That the expected reduction of $\alpha$ synuclein after CLR01 treatment only occurred in the striatum may be due to higher concentrations achieved after ICV infusion in this region. In fact, the doses used in our study were relatively 
low $(1$ and $10 \mu \mathrm{M})$ and chosen based on previous data in zebrafish [26]. Ongoing studies suggest that doses several folds higher than $10 \mu \mathrm{M}$ are safe. However, our data show that using intracerebroventricular administration of CLR01 in the Thy1aSyn model, the higher dose did not further improve the therapeutic effect in the striatum, as more robust improvements were seen with the lower of the 2 doses we used.

An alternative explanation is that CLR01 differently affects $\alpha$-synuclein assembly in different neuronal populations. Strikingly, the morphological aspect of $\alpha$-synuclein aggregates differs in different brain regions of the Thy1-aSyn mice, with large aggregates in the SN [32] and only punctate small aggregates in striatum. This suggests that $\alpha$-synuclein misfolding, aggregation, and/or membrane association differ in the 2 regions and may be differently affected by CLR 01 .

Low brain levels of the drug may have contributed to the more moderate improvements in the challenging beam after $\mathrm{SC}$ infusion relative to the intracerebroventricular administration. Interestingly, in the pole test, subcutaneous treatment led to significant improvement, whereas intracerebroventricular administration did not (see Figs S2 and 4). Compared with the challenging-beam test, the pole test might reflect dysfunction in different brain regions than the beam test, and, as mentioned above, diffusion of the drug through the brain might differ after intracerebroventricular and subcutaneous infusions. Effects on the peripheral nervous system also could be involved. Thyl-aSyn mice show $\alpha$-synuclein accumulation in peripheral neurons and neurites, which would be targeted by subcutaneous administration of CLR01, as has been shown in a mouse model of transthyretin amyloidosis [30] but not by intracerebroventricular administration.

At the young age used here, Thy1-aSyn mice exhibit $\alpha$ synuclein pathology, behavioral deficits and alterations in the corticostriatal circuitry $[32,39]$, whereas progressive loss of dopamine does not occur until 14 months of age in this model. The encouraging effects of CLR01 presented here pave the way for further long-term studies in older Thy1-aSyn mice. Overall, the beneficial effect and safety profile of CLR01 in the Thy1-aSyn mouse model reported here supports future development of this class of compounds for the treatment of $\mathrm{PD}$ and other synucleinopathies.

\begin{abstract}
Acknowledgements This work was supported by National Institutes of Health (NIH)/National Institute of Neurological Disorders and Stroke (NINDS) P50 grant NS38367 [University of California, Los Angeles (UCLA), Morris K. Udall Parkinson Disease Research Center of Excellence], including a Blueprint supplement to this award, NIH/ National Institute of Environmental Health Sciences P01 grant ES016732, RJG Foundation grant 20095024, the Michael J. Fox Foundation, Team Parkinson/Parkinson Alliance, The American Parkinson's Disease Association, and gifts to the Center for the Study of Parkinson's Disease at UCLA.
\end{abstract}

Required Author Forms Disclosure forms provided by the authors are available with the online version of this article.

\section{References}

1. Spillantini MG, Goedert M. The $\alpha$-synucleinopathies: Parkinson's disease, dementia with Lewy bodies, and multiple system atrophy. Ann N Y Acad Sci 2000;920:16-27.

2. Vekrellis K, Stefanis L. Targeting intracellular and extracellular $\alpha$ synuclein as a therapeutic strategy in Parkinson's disease and other synucleinopathies. Expert opinion on therapeutic targets 2012;16(4):421-432.

3. Trojanowski JQ, Lee VM. Parkinson's disease and related $\alpha$ synucleinopathies are brain amyloidoses. Ann N Y Acad Sci 2003;991:107-110.

4. Devine MJ, Gwinn K, Singleton A, Hardy J. Parkinson's disease and $\alpha$-synuclein expression. Mov Disord 2011;26(12):2160-2168.

5. Simon-Sanchez J, Schulte C, Bras JM, et al. Genome-wide association study reveals genetic risk underlying Parkinson's disease. Nat Genet 2009;41(12):1308-1312.

6. Satake W, Nakabayashi Y, Mizuta I, et al. Genome-wide association study identifies common variants at four loci as genetic risk factors for Parkinson's disease. Nat Genet 2009;41(12):1303-1307.

7. Edwards TL, Scott WK, Almonte C, et al. Genome-wide association study confirms SNPs in SNCA and the MAPT region as common risk factors for Parkinson disease. Ann Hum Genet 2010;74(2):97-109.

8. Liu J, Xiao Q, Wang Y, et al. Analysis of genome-wide association study-linked loci in Parkinson's disease of Mainland China. Mov Disord 2013;28(13):1892-1895.

9. Hatami A, Chesselet MF. Transgenic rodent models to study $\alpha$ synuclein pathogenesis, with a focus on cognitive deficits. Curr Top Behav Neurosci 2015;22:303-330.

10. Mizuno H, Fujikake N, Wada K, Nagai Y. $\alpha$-Synuclein transgenic Drosophila as a model of Parkinson's disease and related synucleinopathies. Parkinsons Dis 2010;2011:212706.

11. Fellner L, Wenning GK, Stefanova N. Models of multiple system atrophy. Curr Top Behav Neurosci 2015;22:369-393.

12. Chesselet MF, Richter F. Modelling of Parkinson's disease in mice. Lancet Neurol 2011;10(12):1108-1118.

13. Lulla A, Barnhill L, Bitan G, et al. Neurotoxicity of the Parkinson's disease-associated pesticide ziram is synuclein-dependent in zebrafish embryos. Environ Health Perspect 2016;124:1766-1775.

14. Xilouri M, Brekk OR, Stefanis L. Autophagy and $\alpha$-synuclein: relevance to Parkinson's disease and related synucleopathies. Mov Disord 2016;31(2):178-192.

15. Subramaniam SR, Vergnes L, Franich NR, Reue K, Chesselet MF. Region specific mitochondrial impairment in mice with widespread overexpression of $\alpha$-synuclein. Neurobiol Dis 2014;70:204-213.

16. Breydo L, Wu JW, Uversky VN. $\alpha$-Synuclein misfolding and Parkinson's disease. Biochim Biophys Acta 2012;1822(2):261-285.

17. Amer DA, Irvine GB, El-Agnaf OM. Inhibitors of $\alpha$-synuclein oligomerization and toxicity: a future therapeutic strategy for Parkinson's disease and related disorders. Exp Brain Res 2006;173(2):223-233.

18. Sinha S, Lopes DH, Du Z, et al. Lysine-specific molecular tweezers are broad-spectrum inhibitors of assembly and toxicity of amyloid proteins. J Am Chem Soc 2011;133(42):16958-16969.

19. Attar A, Bitan G. Disrupting self-assembly and toxicity of amyloidogenic protein oligomers by "molecular tweezers"-from the test tube to animal models. Curr Pharm Des 2014;20(15):24692483.

20. Schrader T, Bitan G, Klarner FG. Molecular tweezers for lysine and arginine - powerful inhibitors of pathologic protein aggregation. Chem Commun (Camb) 2016;52(76):11318-11334.

21. Talbiersky P, Bastkowski F, Klarner FG, Schrader T. Molecular clip and tweezer introduce new mechanisms of enzyme inhibition. J Am Chem Soc 2008;130(30):9824-9828. 
22. Acharya S, Safaie BM, Wongkongkathep P, et al. Molecular basis for preventing $\alpha$-synuclein aggregation by a molecular tweezer. $\mathrm{J}$ Biol Chem 2014;289(15):10727-10737.

23. Bier D, Rose R, Bravo-Rodriguez K, et al. Molecular tweezers modulate 14-3-3 protein-protein interactions. Nat Chem 2013;5(3):234-239.

24. Klärner FG, Schrader T. Aromatic interactions by molecular tweezers and clips in chemical and biological systems. Acc Chem Res 2013;46(4):967-978

25. Attar A, Chan WT, Klärner FG, Schrader T, Bitan G. Safety and pharmacological characterization of the molecular tweezer CLR01 - a broad-spectrum inhibitor of amyloid proteins' toxicity. BMC Pharmacol Toxicol 2014;15(1):23.

26. Prabhudesai S, Sinha S, Attar A, et al. A novel "molecular tweezer" inhibitor of $\alpha$-synuclein neurotoxicity in vitro and in vivo. Neurotherapeutics 2012;9(2):464-476.

27. Fogerson SM, van Brummen AJ, Busch DJ, et al. Reducing synuclein accumulation improves neuronal survival after spinal cord injury. Exp Neurol 2016;278:105-115.

28. Busch DJ, Morgan JR. Synuclein accumulation is associated with cell-specific neuronal death after spinal cord injury. J Comp Neurol 2012;520(8):1751-1771.

29. Attar A, Ripoli C, Riccardi E, et al. Protection of primary neurons and mouse brain from Alzheimer's pathology by molecular tweezers. Brain 2012;135(Pt 12):3735-3748.

30. Ferreira N, Pereira-Henriques A, Attar A, et al. Molecular tweezers targeting transthyretin amyloidosis. Neurotherapeutics 2014;11(2): 450-461.

31. Rockenstein E, Mallory M, Hashimoto M, et al. Differential neuropathological alterations in transgenic mice expressing $\alpha$-synuclein from the platelet-derived growth factor and Thy-1 promoters. J Neurosci Res 2002;68(5):568-578.

32. Chesselet MF, Richter F, Zhu C, et al. A progressive mouse model of Parkinson's disease: the Thy1-aSyn ("Line 61") mice. Neurotherapeutics 2012;9(2):297-314.

33. Magen I, Torres ER, Dinh D, et al. Social cognition impairments in mice overexpressing $\alpha$-synuclein under the Thy1 promoter, a model of pre-manifest Parkinson's disease. J Parkinsons Dis 2015;5(3):669-680.

34. McDowell KA, Shin D, Roos KP, Chesselet MF. Sleep dysfunction and EEG alterations in mice overexpressing $\alpha$-synuclein. $J$ Parkinsons Dis 2014;4(3):531-539.

35. Grant LM, Richter F, Miller JE, et al. Vocalization deficits in mice over-expressing $\alpha$-synuclein, a model of pre-manifest Parkinson's disease. Behav Neurosci 2014;128(2):110-121.

36. Watson MB, Richter F, Lee SK, et al. Regionally-specific microglial activation in young mice over-expressing human wildtype $\alpha$-synuclein. Exp Neurol 2012;237(2):318-334.

37. Wang L, Magen I, Yuan PQ, et al. Mice overexpressing wild-type human $\alpha$-synuclein display alterations in colonic myenteric ganglia and defecation. Neurogastroenterol Motil 2012;24(9):e425-e436.

38. Magen I, Fleming SM, Zhu C, et al. Cognitive deficits in a mouse model of pre-manifest Parkinson's disease. Eur J Neurosci 2012;35(6):870-882.

39. Lam HA, Wu N, Cely I, et al. Elevated tonic extracellular dopamine concentration and altered dopamine modulation of synaptic activity precede dopamine loss in the striatum of mice overexpressing human $\alpha$-synuclein. J Neurosci Res 2011;89(7):1091-1102.

40. Paxinos G, Franklin KBJ. The mouse brain in stereotaxic coordinates. San Diego: Academic Press; 2001.

41. Fleming SM, Salcedo J, Fernagut PO et al. Early and progressive sensorimotor anomalies in mice overexpressing wild-type human $\alpha$-synuclein. J Neurosci 2004;24(42):9434-9440.
42. Richter F, Fleming SM, Watson M, et al. A GCase chaperone improves motor function in a mouse model of synucleinopathy. Neurotherapeutics 2014;11(4):840-856.

43. Fleming SM, Mulligan CK, Richter F, et al. A pilot trial of the microtubule-interacting peptide (NAP) in mice overexpressing $\alpha$ synuclein shows improvement in motor function and reduction of $\alpha$-synuclein inclusions. Mol Cell Neurosci 2011;46(3):597-606.

44. Magen I, Ostritsky R, Richter F, et al. Intranasal NAP (davunetide) decreases tau hyperphosphorylation and moderately improves behavioral deficits in mice overexpressing $\alpha$-synuclein. Pharmacol Res Perspect 2014;2:e0065.

45. Shankar GM, Li S, Mehta TH, et al. Amyloid- $\beta$ protein dimers isolated directly from Alzheimer's brains impair synaptic plasticity and memory. Nat Med 2008;14(8):837-842.

46. Abramoff MD, Magelhaes PJ, Ram SJ. Image processing with ImageJ. Biophotonics Intl 2004;11(7):36-42.

47. Lopes DH, Sinha S, Rosensweig C, Bitan G. Application of photochemical cross-linking to the study of oligomerization of amyloidogenic proteins. Methods Mol Biol 2012;849:11-21.

48. Richter F, Gao F, Medvedeva V, et al. Chronic administration of cholesterol oximes in mice increases transcription of cytoprotective genes and improves transcriptome alterations induced by $\alpha$ synuclein overexpression in nigrostriatal dopaminergic neurons. Neurobiol Dis 2014;69:263-275.

49. Fernagut PO, Hutson CB, Fleming SM, et al. Behavioral and histopathological consequences of paraquat intoxication in mice: effects of $\alpha$-synuclein over-expression. Synapse 2007;61(12):991-1001.

50. Efron B, Tibshirani R. Statistical data analysis in the computer age. Science 1991;253(5018):390-395.

51. Rabl R, Breitschaedel C, Flunkert S, et al. Early start of progressive motor deficits in Line $61 \alpha$-synuclein transgenic mice. BMC Neurosci 2017;18(1):22.

52. Friden M, Ljungqvist $\mathrm{H}$, Middleton B, Bredberg U, HammarlundUdenaes $M$. Improved measurement of drug exposure in the brain using drug-specific correction for residual blood. J Cereb Blood Flow Metab 2010;30(1):150-161.

53. Dagenais C, Rousselle C, Pollack GM, Scherrmann JM. Development of an in situ mouse brain perfusion model and its application to mdrla P-glycoprotein-deficient mice. J Cereb Blood Flow Metab 2000;20(2):381-386.

54. Oddo S, Caccamo A, Shepherd JD, et al. Triple-transgenic model of Alzheimer's disease with plaques and tangles: intracellular $A \beta$ and synaptic dysfunction. Neuron 2003;39(3):409-421.

55. Franich NR, Mallajosyula JK, Richter F, et al. Reducing $\alpha$ synuclein after deficit onset improves behavioral deficits and pathology in over-expressing mice. Neurosci Abstr 2013;622.26/I18.

56. Levine MS, Cepeda C, Hickey MA, Fleming SM, Chesselet MF. Genetic mouse models of Huntington's and Parkinson's diseases: illuminating but imperfect. Trends Neurosci 2004;27(11):691-697.

57. Hickey MA, Zhu C, Medvedeva V, et al. Evidence for behavioral benefits of early dietary supplementation with CoEnzymeQ10 in a slowly progressing mouse model of Huntington's disease. Mol Cell Neurosci 2012;49(2):149-157.

58. Rockenstein E, Nuber S, Overk CR, et al. Accumulation of oligomer-prone $\alpha$-synuclein exacerbates synaptic and neuronal degeneration in vivo. Brain 2014;137(Pt 5):1496-1513.

59. Rahimi F, Bitan G. The structure and function of fibrillar and oligomeric assemblies of amyloidogenic proteins. In: Rahimi F, Bitan G, Eds. Non-fibrillar amyloidogenic protein assemblies - common cytotoxins underlying degenerative diseases. Dordrecht: Springer Science + Media B.V.; 2012. p. 1-36.

60. Bitan G, Fradinger EA, Spring SM, Teplow DB. Neurotoxic protein oligomers - what you see is not always what you get. Amyloid 2005;12(2):88-95. 